\title{
Research on Hierarchical Storage System based on Wireless Mesh Network
}

\author{
Xin $\mathrm{Hu}$ \\ Library, Nanchang Institute of Technology, Jiang Xi 330013, China \\ huxii@163.com
}

Keywords: Storage, NVRAM, SSD, HDD.

\begin{abstract}
This thesis according to this hot technique of Wireless Mesh Network day by day mature background, on the foundation of comprehending the basic theories of Wireless Mesh Network, we emphasizes a rightness how to keep enough bandwidth and band rate in much jump of the Mesh environment in certain analysis and research. Through the experiment test and carried on certain data analysis, put forward of construct a kind of new structure to solve this problem. Take the applied aspect of Wireless Mesh Network, we combine the point of this text research problem and the construction present condition of wireless city in the home and abroad, elaborate outstanding position of Wireless Mesh Network in the construction of wireless city and how to construct a wireless city. Explain the important meaning of Wireless Mesh Network in the next generation wireless network.
\end{abstract}

\section{Introduction}

In recent years, along with more and more the deployment of the large-scale wireless network Wireless Mesh Network (WMN) is more and more valued by people, especially the emergence of Wireless Mesh Network satisfied a social demand more [1], its conduct and actions a kind of new network structure, just become a hot point in the research of next generation wireless access.

Nowadays, the development trend of storage systems tends to be of mass storage, low cost and high performance, while any kind of storage device, such as Non-Volatile Random Access Memory (NVRAM), Solid State Disk (SSD) and Hard Disk Drive (HDD) [2-3], cannot meet the needs of these demands mentioned above due to its intrinsic characteristics. Hybrid storage systems, built by making the most of the properties of different types of storage devices, are not only capable to substantially extend the storage capacity, but also able to remarkably improve the performance while keeping a low system cost. Therefore, hybrid storage is becoming the development direction of storage technology.

But people discover the Wireless Mesh Network exists some baffling when large-scale deployed in actual, especially multiple hops problem, this problem cause of bandwidth degradation, radio interference and network latency. etc., these problems make the performance of Wireless Mesh Network descend a lot.

\section{Organization of the Text}

In this thesis, the concept, development history and challenges of hybrid storage systems are introduced, based on which a hybrid storage system named Trident is designed and implemented. The thesis is focused on the following aspects:

(1)The development history of hybrid storage systems is studied and a deep analysis on the critical technology problems in hybrid storage system design is made.

(2)Trident, a hybrid storage system based on NVRAM, SSD and HDD is designed and implemented, the issues of which includes architecture, mapping policy, hot data identification, data migration and so on.

(3)Synthetic workload and production workload are used to test and compare the random and sequential access performance of HDD-only, Facebook Flash Cache, and Trident storage systems. 
The cache hit rates are also compared between Flash Cache and Trident systems. The test results show that, to the Trident system, the sequential access performance is close to that of HDD-only storage system, the IOPS partially exceeds that of Flash Cache, and the cache hit rate is close to that of Flash Cache with fewer write to SSD compare to Flash Cache. This system is implemented as a kernel module in the Linux OS and is transparent to upper layer applications. The research achievement of this thesis provides a reference for the design and implementation of other hybrid storage systems.

\section{Design process}

According to the shortage of WLAN, this paper studied a architecture of wireless mesh network, which has wide applicability. In this paper, the wireless Mesh AP based on embedded system mentioned has compatibility and interoperability [4]. Clinux system is set up on embedded board JA76PF0. According to the design demand, on the platform realizes the wireless Mesh AP node function, wireless network card driver, network management and the security of the wireless Mesh AP. By wireless network driver program Madwifi, set the two wireless card for different working mode. So the wireless Mesh backbone network can connect to the client network. Through software download, configuration files modified and compile the transplant of hostapd daemon, network address translation, dynamic host allocation protocol, network time protocol. Realized wireless parameter configuration and management and the reasonable use of network resources in the process of the network. By ESS1D hidden, MAC address filtering technology and WPA encryption authentication technology, the wireless Mesh AP is safe in the process of network. (Fig. 1 and Table 1)

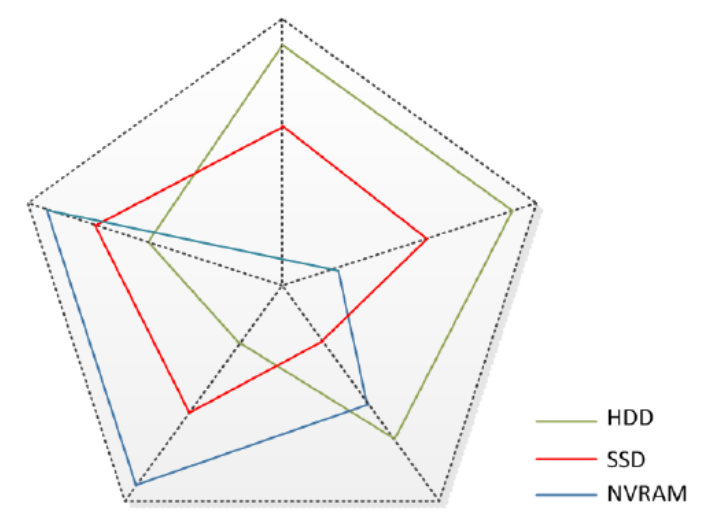

Fig. 1 Characteristics differences among several storage devices

Table 1 compared with NVRAM/ SSD/ HDD

\begin{tabular}{cccc}
\hline NVRAM & FRAM & $55 \mathrm{NS}$ & $10 \mathrm{MB}$ \\
\hline SSD & FLASH-BASED SSD & $25 \mathrm{US}$ & $100 \mathrm{~GB}$ \\
HDD & SATA & $8.5 \mathrm{MS}$ & $1000 \mathrm{~GB}$ \\
SSD & BBDRAM & $70-100 \mathrm{NS}$ & $1 \mathrm{~GB}$ \\
\hline
\end{tabular}

Once again, the AODV routing protocol is successfully portal to the embedded board and according to the actual need to study a improved AODV routing protocol based on the parameters of wireless communication link stability [5]. By modying source code, data structure of Nettilter, and hook function, realized transplant of AODV routing protocol. Trough testing the stability of the wireless communication link and routing based on the stability of wireless communication link, realized improved routing protocol. By the simulation test of AODV and improved AODV in NS, proved improved AODV is better at network throughput and packet loss rate than AODV. [6] Finally, we designed wireless Mesh AP configuration and management platform based on B/S. By set up Web server on embedded board JA76PF0 and the interaction of Web server with CGI, we can read anct configure the parameter in long. Trough function, system function and shell script command, we can read and configure the parameter of wireless Mesh AI. By html, JavaScript script and CSS, an 
interface is realized. After repeated origination test, the response time is less than 0.4 seconds. (Fig. 2 and 3)

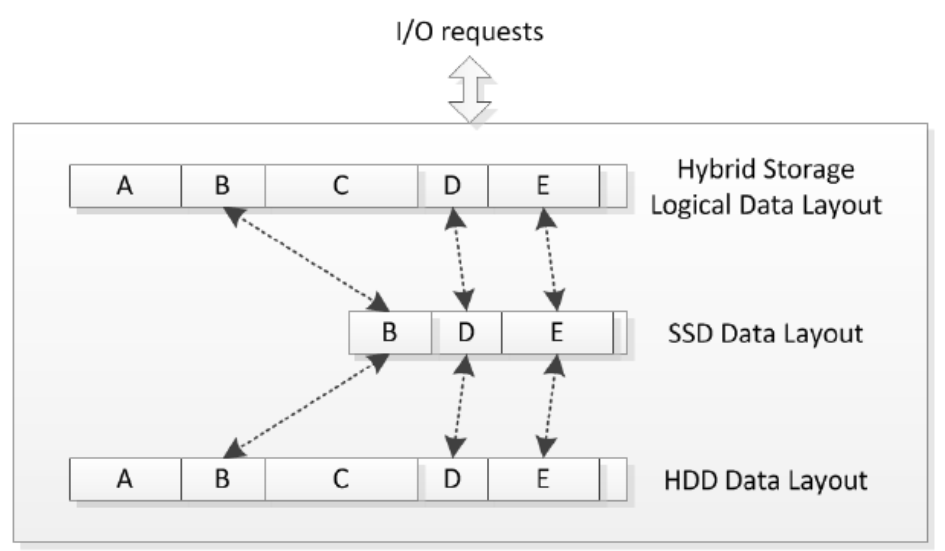

Fig. 2 Multi-layer cache architecture I/O requests

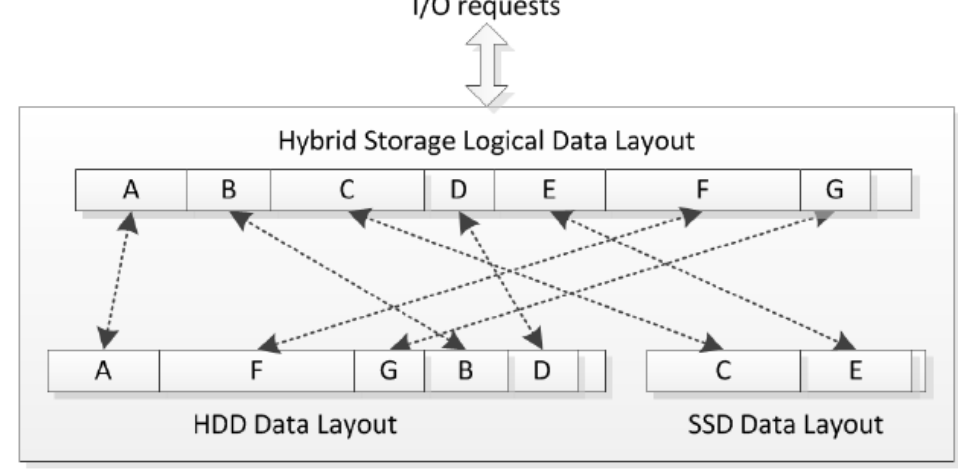

Fig. 3 Single-layer architecture

\section{Key technology}

Nowadays distributed management methods of wired network mainly points for the stable environment with width stability, performance nodes. The methods will not continuous services, and scalability and high complexity dynamicity of mobile environments with be adaptable for the mobile data management heterogeneity, distributed, high dimensions, and immovability, and so they doesn't be directly applied into data management in mobile environment. For the multi-sources, multi-sinks, self-control, context aware and environment dependability, and the centralization and distribution coexistence of mobile environment oriented data storage management, there are many technologies which may be combined with to construct mobile environment storage management and service system by mobile agent, mobile database, distributed network, cross layer cooperation, grid computing, cloud computing and etc. Furthermore, along with the internet work and convergence technology becoming adult, mobile environmental devices may be cooperated with grid computing and cloud computing system to construct massive, persistent, boundless scalable storage resources and service system, which will bring about huge market prospect, and good data storage management methods will provide high efficient, secure data storage foundation. Consequently, mobile environment oriented data storage management methods will show important theoretical and practical meanings. Shown in Eq. 1.

$$
\text { target set }=\frac{d b n}{\text { block size } * \text { set size }} \bmod (\text { number of sets })
$$

Organizing data in a reasonable, effective and dependable way is a key problem to P2P storage systems. The original DHT are designed to treat peers equally. Thus yields good load balancing and robustness to failures. However, with the development of the system the weak peers can seriously compromise the scalability and efficiency of the whole system. So we present a novel hierarchical DHT routing protocol which is built on Chord but achieves better file routing efficiency in Internet by 
exploiting heterogeneity and proximity. 'This protocol exploits the proximity by grouping the data with the same properties together and distributing the data across peers within a matching cluster, which greatly boosts the local access ratio of data and optimizes the routing performance of our protocol. (Fig. 4)

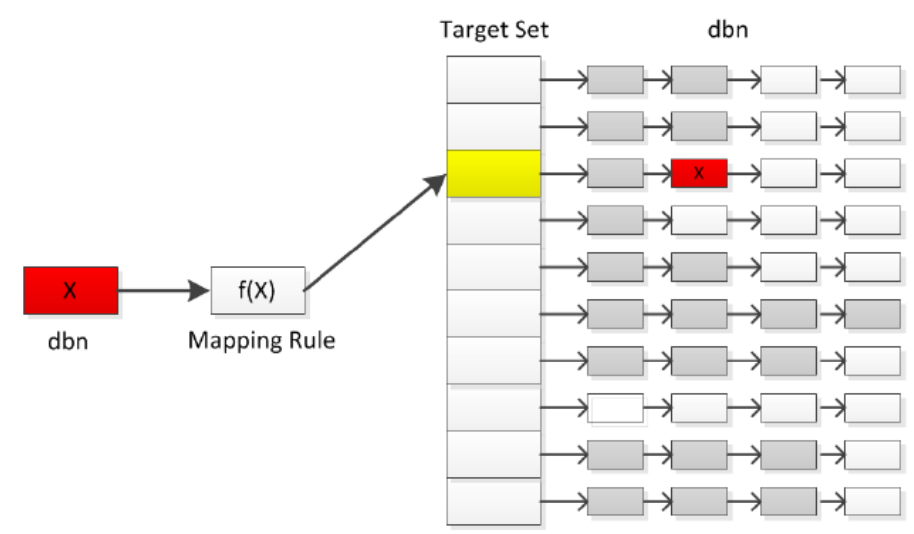

Fig. 4 The block based group mapping in FlashCache

DHT network needs relatively higher maintenance overhead than unstructured P2P networks. As result from building and updating their routing information, p2p system generates considerable maintenance workload which not only increases the bandwidth consumption but also affects the routing efficiency. So we present here a new maintenance method to minimize the maintenance cost in a large-scale system or a dynamic environment which combines the hierarchical DHT algorithm with a peer proximity mechanism. We let storage peer in the lower layer periodically probe their successors. Shown in Eq. 2.

$$
\mathrm{RC}\left(E_{c}, \mathrm{D}\right)=\frac{\text { Capacity required by extent }}{\text { Total space in device }}
$$

Parallel download tools, such as Bit Torrent, are very popular and scalable file distribution systems which can distribute large files quickly and efficiently however, those systems based on tracker server relies on static IP address. Aiming at this issue, this dissertation proposed a dynamic parallel downloading method Based on hierarchical DHT. The method couples the unique ID of a tracker server with the ID of its resident manage peer, and introduces distributed ID mapping service to implement correct route in dynamic Internet environment. By the combination of grouping peers and the locality of the downloading, the method optimizes the management of transferring and improves downloading performance greatly.

\section{Conclusions}

A model of hierarchical storage system based on wireless mesh network (HSSWMN, Hierarchical Storage System over Wireless Mesh Network) is proposed. Firstly, the dissertation proposes the hierarchical storage system model based on wireless mesh network (HSSWMN), and the storage model, access algorithms and performance optimization methods are analyzed and researched. Secondly, name space and metadata service, search and looking for service, registration and logout, scalability, load balance, fault tolerant, data safety, replication and cache mechanism, topology reconstruction of HSSWMN are researched. At last, by the simulation analysis, simulation testing discloses that the delay, throughput, error code and etc, performance analysis is carried for the feasibility, availability and reliability of HSSWMN storage system.

\section{References}

[1] M. Satyanarayanan, J. J. Kistler, P. Kumar. Coda: a Highly Available File System for a Distributed Workstation Environment. IEEE Transactions on Computers, 2009., 6(4): 715-726. 
[2] Kuhn Michael, Kunkel Julian Martin, Ludwig Thomas. Dynamic file system semantics to enable metadata optimizations in PVFS. Concurrency Computation Practice and Experience, September 25, 2009, 21(14): 1775-1788.

[3] Information on http: //www. napster. com

[4] A. Rowstron and P. Druschel. Pastry: Scalable, distributed object location and routing for large-scale peer-to-peer systems. IFIP/ACM International Conference on Distributed Systems Platforms(Middleware), Heidelberg, Germany, pages November, 2011: 329-350.

[5] Information on http: //www. w3. org/XML

[6] Information on http: //www. w3. org/RDF 